\title{
DIS and the effects of fluctuations: a momentum space analysis
}

\author{
E. Basso $^{\text {a }}$, M.B. Gay Ducati ${ }^{\mathrm{b}}$, E.G. de Oliveira $^{\mathrm{c}}$, J.T. de Santana Amaral ${ }^{\mathrm{d}}$ \\ Instituto de Física, Universidade Federal do Rio Grande do Sul, Caixa Postal 15051, 91501-970 Porto Alegre, RS, Brazil
}

Received: 17 July 2008 / Published online: 26 September 2008

(C) Springer-Verlag / Società Italiana di Fisica 2008

\begin{abstract}
Among the dipole models of deep inelastic scattering at small values of the Bjorken variable $x$, one has been recently proposed which relates the virtual photonproton cross section to the dipole-proton forward scattering amplitude in momentum space. The latter is parametrized by an expression which interpolates between its behavior at saturation and the travelling wave, ultraviolet, amplitudes predicted by perturbative QCD from the BalitskyKovchegov equation. Inspired by recent developments in coordinate space, we use this model to parametrize the structure function of the proton and confront it with HERA data on ep deep inelastic scattering. Both event-by-event and physical amplitudes are considered, the latter being used to investigate the effect of gluon number fluctuations, beyond the mean-field approximation. We conclude that fluctuations are not present in DIS at HERA energies.
\end{abstract}

\section{Introduction}

The well known correspondence between the evolution in QCD at small- $x$ (at high energy) and a reaction-diffusion process has been the main source of recent knowledge concerning the scattering amplitudes and their evolution towards the high energy limit. In particular, it has been realized that the Balitsky-JIMWLK equations do not take into account the influence of the gluon number fluctuations [14]. In the mean-field approximation, these equations reduce to a unique equation, the so-called Balitsky-Kovchegov (BK) equation [5-7], the simplest equation that describes the evolution of the dipole scattering amplitude with rapidity $Y \equiv \ln 1 / x$. Being a mean-field version of the BalitskyJIMWLK equations, the BK equation does not include the effects of discreteness and consequently of the fluctuations. Among the consequences of the fluctuations, at least in the

\footnotetext{
a e-mail: andre.basso@ufrgs.br

b e-mail: beatriz.gay@ufrgs.br

c e-mail: emmanuel.deoliveira@ufrgs.br

d e-mail: thiago.amaral@ufrgs.br
}

fixed coupling case, one can cite, for example, the slowing down of the approach towards the unitarity limit, as compared to the mean-field framework, and the break down of geometric scaling [8], a phenomenological feature observed at the DESY ep collider HERA, in measurements of inclusive $\gamma^{*} p$ scattering, which is naturally explained in terms of the traveling wave solutions of the BK equation [9-11].

At small $x$, electron-proton deep inelastic scattering (DIS) can be seen in a particular frame, called the $d i$ pole frame, which allows for the factorization of the virtual photon-proton cross section. In this frame, the proton carries most of the total energy, but the photon has enough energy to split into a quark-antiquark pair, or a dipole. This $q \bar{q}$ pair then interacts with the proton. The virtual photonproton cross section can be written as

$\sigma_{T, L}^{\gamma^{*} p}\left(Q^{2}, Y\right)=\int \mathrm{d}^{2} r \int_{0}^{1} \mathrm{~d} z\left|\Psi_{T, L}\left(\boldsymbol{r}, z ; Q^{2}\right)\right|^{2} \sigma_{\mathrm{dip}}(\boldsymbol{r}, Y)$,

where the labels $T$ and $L$ refer, respectively, to the transverse and longitudinal parts of the cross section, $\boldsymbol{r}=\boldsymbol{x}-\boldsymbol{y}$ is the vector which gives the transverse size of the dipole, $\boldsymbol{x}$ and $\boldsymbol{y}$ being the transverse coordinates of the quark and the antiquark; $z$ is the fraction of the momentum of the photon carried by the quark and $\Psi_{T, L}\left(\boldsymbol{r}, z ; Q^{2}\right)$ are the transverse and longitudinal wave functions for the photon to go into the dipole, whose explicit forms are well known from QED. Using (1) one can obtain the expression for the $F_{2}$ proton structure function through the formula

$F_{2}\left(x, Q^{2}\right)=\frac{Q^{2}}{4 \pi^{2} \alpha_{\mathrm{em}}}\left[\sigma_{T}^{\gamma^{*} p}\left(x, Q^{2}\right)+\sigma_{L}^{\gamma^{*} p}\left(x, Q^{2}\right)\right]$.

The quantity $\sigma_{\text {dip }}$ in (1) is the dipole-proton cross section which can be expressed as

$\sigma_{\operatorname{dip}}^{\gamma^{*} p}(\boldsymbol{r}, Y)=2 \int \mathrm{d}^{2} \boldsymbol{b}\langle T(\boldsymbol{r}, \boldsymbol{b})\rangle_{Y}$,

where $\langle T(\boldsymbol{r}, \boldsymbol{b})\rangle_{Y}$ is the average scattering amplitude-the notation $\langle\cdot\rangle$ denotes the average over all the realizations of 
the target (proton) color field-for the dipole-proton scattering at a given impact parameter $\boldsymbol{b}=(\boldsymbol{x}+\boldsymbol{y}) / 2$.

As the explicit forms of the virtual photon wave functions are well known, one is left with the parametrization of the scattering amplitude, that is, one has to model the dipole-proton cross section. Different approaches have already been proven successful, giving a good description of the data; among them one can cite the model proposed by Golec-Biernat and Wusthoff, the GBW saturation model [12], and the model by Iancu, Itakura and Munier, the IIM model or CGC fit [13], both developed in coordinate space. These models have recently been considered in order to study the effects of fluctuations on DIS in the fixed coupling case [14]. Although the description of the DIS data is improved once gluon number fluctuations are included, it is not possible to state that the improvements come from them, but they may come from the geometric scaling violation present, for example, in the diffusion part of BK solution present in the IIM model.

In this work we perform an analysis similar to that done in [14] by using a recently proposed parametrization for the dipole-proton scattering amplitude, the first developed in momentum space [15].

In Sect. 2 we present a review on the main features of the QCD evolution at high energies within the dipole picture [16-18]. Section 3 is devoted to a description of the parametrization for the scattering amplitude in momentum space that will be used to describe the DIS data. The fluctuations are properly included, the average amplitude is defined and the results of the fit to the last HERA data are presented. A discussion and conclusions are presented in Sect. 4.

\section{The dipole scattering amplitude}

\subsection{Dipole evolution}

Considering a dipole of transverse coordinates $\boldsymbol{x}$ and $\boldsymbol{y}$ at a given rapidity $Y$, if one increases the rapidity from $Y$ to $Y+\delta Y$, there is a probability for a gluon with transverse coordinate $z$ to be emitted by the quark (or antiquark) of the pair. In the large $N_{\mathrm{c}}$ limit ( $N_{\mathrm{c}}$ is the number of colors), this gluon can be considered as a quark-antiquark pair-a new dipole-at point $z$. This is the dipole picture introduced by Mueller [16-18].

The probability density for the original dipole to split into the two child dipoles $(\boldsymbol{x}, \boldsymbol{z})$ and $(\boldsymbol{z}, \boldsymbol{y})$ is given by

$$
\frac{\bar{\alpha}}{2 \pi} \mathcal{M}(\boldsymbol{x}, \boldsymbol{y}, \boldsymbol{z}) \mathrm{d} Y \mathrm{~d}^{2} z,
$$

where $\bar{\alpha}=\alpha_{\mathrm{s}} N_{\mathrm{c}} / \pi$ and

$$
\mathcal{M}(\boldsymbol{x}, \boldsymbol{y}, \boldsymbol{z})=\frac{(\boldsymbol{x}-\boldsymbol{y})^{2}}{(\boldsymbol{x}-\boldsymbol{z})^{2}(z-\boldsymbol{y})^{2}} .
$$

If the target (in this case, the proton) is dense enough, both child dipoles can interact with it and the resulting evolution equation for the dipole scattering amplitude is [5]

$$
\begin{aligned}
\partial_{Y}\langle T(\boldsymbol{x}, \boldsymbol{y})\rangle= & \bar{\alpha} \int \mathrm{d}^{2} z \mathcal{M}(\boldsymbol{x}, \boldsymbol{y}, \boldsymbol{z})[\langle T(\boldsymbol{x}, \boldsymbol{z})\rangle+\langle T(\boldsymbol{z}, \boldsymbol{y})\rangle \\
& -\langle T(\boldsymbol{x}, \boldsymbol{y})\rangle-\langle T(\boldsymbol{x}, \boldsymbol{z}) T(\boldsymbol{z}, \boldsymbol{y})\rangle] .
\end{aligned}
$$

This is not a closed equation for the one-dipole scattering amplitude, but the first equation of an infinite hierarchy, the Balitsky-JIMWLK hierarchy [5, 19-21]. The first and second terms give the contribution of each dipole which can interact with the proton; the third term gives the virtual contributions to the scattering and the last suppression (quadratic) term comes from taking into account multiple interactions, when both child dipoles interact with the target. When $T$ is small, this quadratic term can be neglected and (6) reduces to the (dipole version of) the linear BFKL equation [22-24].

\subsection{Balitsky-Kovchegov equation}

In the mean-field approximation, valid when the target is sufficiently large and homogeneous, one can write

$\langle T(\boldsymbol{x}, \boldsymbol{z}) T(\boldsymbol{z}, \boldsymbol{y})| \approx\langle T(\boldsymbol{x}, \boldsymbol{z})\rangle\langle T(\boldsymbol{z}, \boldsymbol{y})\rangle$

and the resulting equation is the so-called BK (BalitskyKovchegov) equation [5-7]:

$$
\begin{aligned}
\partial_{Y}\langle T(\boldsymbol{x}, \boldsymbol{y})\rangle= & \bar{\alpha} \int \mathrm{d}^{2} z \mathcal{M}(\boldsymbol{x}, \boldsymbol{y}, \boldsymbol{z})[\langle T(\boldsymbol{x}, \boldsymbol{z})\rangle+\langle T(\boldsymbol{z}, \boldsymbol{y})\rangle \\
& -\langle T(\boldsymbol{x}, \boldsymbol{y})\rangle-\langle T(\boldsymbol{x}, \boldsymbol{z})\rangle\langle T(\boldsymbol{z}, \boldsymbol{y})\rangle] .
\end{aligned}
$$

This equation includes unitarity corrections and is free from the problem of diffusion to the infrared (non-perturbative) region, present in the solution of BFKL equation, since there is a scale, the saturation scale $Q_{\mathrm{s}}(Y)$, an increasing function of rapidity, which naturally emerges from the BK equation.

If one neglects the dependence on the impact parameter, the scattering amplitude depends only on the size of the dipole and (7) becomes an equation for $\langle T(r)\rangle$, where $r=|\boldsymbol{r}|=|\boldsymbol{x}-\boldsymbol{y}|$. Let us denote this mean-field amplitude by $\mathcal{N}_{Y}(r)$. After performing the Fourier transform

$$
N_{Y}(k)=\frac{1}{2 \pi} \int \frac{\mathrm{d}^{2} r}{r^{2}} \mathrm{e}^{i \mathbf{k} \cdot \mathbf{r}} \mathcal{N}_{Y}(r)=\int_{0}^{\infty} \frac{\mathrm{d} r}{r} J_{0}(k r) \mathcal{N}_{Y}(r),
$$

one gets the $\boldsymbol{b}$-independent BK equation in momentum space:

$$
\begin{aligned}
\partial_{Y} N_{Y}(k)= & \frac{\bar{\alpha}}{\pi} \int \frac{\mathrm{d} p^{2}}{p^{2}}\left[\frac{p^{2} N_{Y}(p)-k^{2} N_{Y}(k)}{\left|k^{2}-p^{2}\right|}\right. \\
& \left.+\frac{k^{2} N_{Y}(k)}{\sqrt{4 p^{4}+k^{4}}}\right]-\bar{\alpha} N_{Y}^{2}(k),
\end{aligned}
$$


which can be rewritten as

$\partial_{Y} N_{Y}=\bar{\alpha} \chi\left(-\partial_{L}\right) N_{Y}-\bar{\alpha} N_{Y}^{2}$,

where

$\chi(\gamma)=2 \psi(1)-\psi(\gamma)-\psi(1-\gamma)$

is the BFKL kernel and $L=\log \left(k^{2} / k_{0}^{2}\right)$, with $k_{0}$ some fixed soft scale.

The kernel (11) can be written with the help of a series and it has been shown that after a saddle point approximation and a change of variables [9-11] the BK equation reduces to the Fisher and Kolmogorov-Petrovsky-Piscounov (FKPP) equation [25, 26], which admits of so-called traveling wave solutions. In QCD language, this means that, at asymptotic rapidities, the scattering amplitude depends only on a single variable, the ratio $k^{2} / Q_{\mathrm{s}}^{2}(Y)$, instead of depending separately on $k^{2}$ and $Y$. This scaling property is called geometric scaling and has been observed in measurements of the proton structure function at HERA [8]. The amplitude is a wavefront which interpolates between 0 and 1 and travels towards large values of $k^{2}$ with speed $\lambda$-the saturation exponent-without deformation. The position of the front, for which $N_{Y}=\mathcal{O}(1)$ is given by the saturation momentum $Q_{\mathrm{s}}(Y)$ or, more specifically, by $\log \left(Q_{\mathrm{s}}^{2}(Y) / k_{0}^{2}\right)=\lambda Y$.

The expression for the tail of the scattering amplitude reads

$$
\begin{aligned}
N_{Y}(k) \stackrel{k \gg Q_{\mathrm{s}}}{\approx}\left(\frac{k^{2}}{Q_{\mathrm{s}}^{2}(Y)}\right)^{-\gamma_{\mathrm{c}}} \log \left(\frac{k^{2}}{Q_{\mathrm{s}}^{2}(Y)}\right) \\
\quad \times \exp \left[-\frac{\log ^{2}\left(k^{2} / Q_{\mathrm{s}}^{2}(Y)\right)}{2 \bar{\alpha} \chi^{\prime \prime}\left(\gamma_{\mathrm{c}}\right) Y}\right],
\end{aligned}
$$

where

$\lambda=\min \bar{\alpha} \frac{\chi(\gamma)}{\gamma}=\bar{\alpha} \frac{\chi\left(\gamma_{\mathrm{c}}\right)}{\gamma_{\mathrm{c}}}=\bar{\alpha} \chi^{\prime}\left(\gamma_{\mathrm{c}}\right)$.

One can observe that the last term in (12) has an important role to play, since it introduces an explicit dependence on rapidity and hence violates geometric scaling. Then, geometric scaling is obtained for

$\log \left(k^{2} / Q_{\mathrm{s}}^{2}(Y)\right) \lesssim \sqrt{2 \chi^{\prime \prime}\left(\gamma_{\mathrm{c}}\right) \bar{\alpha} Y}$,

i.e., within a window $\sqrt{Y}$ above the saturation scale.

\subsection{Beyond the mean-field approximation: the effects of fluctuations}

The BK equation is the simplest equation which describes high energy dipole evolution and scattering in perturbative QCD. This mean-field equation has been shown to be in the universality class of the FKPP equation, whose dynamics is called reaction-diffusion dynamics. Within this correspondence between a reaction-diffusion process and the QCD evolution at high energy, it has recently been realized that the Balitsky-JIMWLK hierarchy is not complete because they do not take into account the gluon (dipole) number fluctuations, which are related to discreteness in the evolution, and thus they are completely missed by the BK equation.

As we shall see in the following, the fluctuations influence dramatically the QCD evolution at high energies, and so the properties of the scattering amplitudes. Their inclusion results in a new hierarchy of evolution equations, the pomeron loop equations [2]. The first equation of this hierarchy is exactly the same as (6), but as one goes to the second one, the evolution equation for the two-dipole amplitude $\left\langle T^{(2)}\right\rangle \equiv\langle T T\rangle$, besides the linear (BFKL) term and the non-linear term, responsible for unitarity corrections, there is a new term, proportional to the one-dipole amplitude $\langle T\rangle$, which is the fluctuation term. More generally, the equation for the $k$-dipole amplitude $\left\langle T^{(k)}\right\rangle$ depends on $\left\langle T^{(k)}\right\rangle,\left\langle T^{(k+1)}\right\rangle$ and $\left\langle T^{(k-1)}\right\rangle$, the latter being the contribution of the fluctuation. After an approximation [2] to get rid of the impact-parameter dependence, this can be written as a Langevin equation for the event-by-event amplitude which is formally the BK equation with a noise term, which lies in the same universality class as the stochastic FKPP equation (sFKPP).

Each realization of the noise means a single realization of the target in the evolution and leads to an amplitude for a single event. Different realizations of the target lead to dispersion of the solutions, and then in the saturation momentum $\rho_{\mathrm{s}} \equiv \ln \left(Q_{\mathrm{s}}^{2} / k_{0}^{2}\right)$ from one event to another. The saturation scale is now a random variable whose average value is given by

$\left\langle Q_{\mathrm{s}}^{2}(Y)\right\rangle=\exp \left[\lambda^{*} Y\right]$

The dispersion in the position of the individual fronts is given by

$\sigma^{2}=\left\langle\rho_{\mathrm{s}}^{2}\right\rangle-\left\langle\rho_{\mathrm{s}}\right\rangle^{2}=D \bar{\alpha} Y$.

The diffusion coefficient $D$, as well as the average saturation exponent $\lambda^{*}$, is analytically known only in the asymptotic limit $\alpha_{\mathrm{s}}^{2} \rightarrow 0$; thus, in what follows they will be treated as free parameters.

The probability distribution of $\rho_{\mathrm{S}}$ is, to a good approximation, Gaussian [27]:

$P_{Y}\left(\rho_{\mathrm{s}}\right) \simeq \frac{1}{\sqrt{\pi \sigma^{2}}} \exp \left[-\frac{\left(\rho_{\mathrm{s}}-\left\langle\rho_{\mathrm{s}}\right\rangle\right)^{2}}{\sigma^{2}}\right]$.

For each single event, the evolved amplitude shows a traveling wave pattern, which means that geometric scaling is preserved for each realization of the noise. However, the speed 
of the wave is smaller than the speed predicted by the BK equation. This speed, or the (average) saturation exponent, has been found to be [2]

$\lambda^{*} \simeq \lambda-\frac{\pi^{2} \gamma_{\mathrm{c}} \chi^{\prime \prime}\left(\gamma_{\mathrm{c}}\right)}{\ln \left(1 / \alpha_{\mathrm{s}}^{2}\right)}$.

The average amplitude is determined by $\left(X \equiv \ln \left(1 / r^{2} Q_{0}^{2}\right)\right)$

$\left\langle T\left(X, \rho_{\mathrm{s}}\right)\right\rangle=\int_{-\infty}^{+\infty} \mathrm{d} \rho_{\mathrm{s}} P_{Y}\left(\rho_{\mathrm{s}}\right) T\left(X, \rho_{\mathrm{s}}\right)$.

A crucial property of the physical amplitudes is that at sufficiently high energies, unlike the individual fronts, they will generally not show geometric scaling. More specifically, they will show additional dependencies on $Y$, through the front dispersion $\sigma$. Then geometric scaling is washed out and replaced by the so-called diffusive scaling $[2-4,28]$

$\left\langle T\left(X, \rho_{\mathrm{s}}\right)\right\rangle=\mathcal{T}\left(\frac{X-\left\langle\rho_{\mathrm{s}}\right\rangle}{\sqrt{\bar{\alpha} D Y}}\right)$.

\section{Description of DIS data}

We have seen in the Introduction that in the dipole frame the structure function of the proton, $F_{2}$, can be written in terms of the dipole-proton cross section $\sigma_{\mathrm{dip}}$, which can be expressed through the average dipole-proton scattering amplitude through (3). If one treats the proton as an homogeneous disk of radius $R_{p}$, i.e., if one neglects the impact-parameter dependence, the amplitude depends only on the size $r$ of the dipole and after integrating out the remaining angular dependence, the dipole-proton cross section can be written in terms of the amplitude $\langle T(r)\rangle$ in the following way:

$\sigma_{\text {dip }}^{\gamma^{*} p}(r, Y)=2 \pi R_{p}^{2}\langle T(r)\rangle$.

This expression must be inserted in (1) and one has to parametrize the dipole scattering amplitude $\langle T(r)\rangle$ in order to the reproduce $\sigma^{\gamma^{*} p}$ measurements. From now on we shall denote this amplitude by $T(r, Y)$.

\subsection{AGBS model and fluctuations}

The most recent parametrization for the dipole-proton scattering amplitude has been proposed by Amaral, Gay Ducati, Betemps and Soyez, the AGBS model [15], which is the first parametrization in momentum space in the literature; it is based on the knowledge of the asymptotic behavior of the solutions of the BK equation. The starting point is that, after performing the Fourier transform (8), it is possible to rewrite the cross section (1) in terms of the amplitude in momentum space. The structure function $F_{2}$ takes the form [15]

$$
\begin{aligned}
& F_{2}\left(x, Q^{2}\right) \\
& \quad=\frac{Q^{2} R_{p}^{2} N_{\mathrm{c}}}{4 \pi^{2}} \int_{0}^{\infty} \frac{\mathrm{d} k}{k} \int_{0}^{1} \mathrm{~d} z\left|\tilde{\Psi}\left(k^{2}, z ; Q^{2}\right)\right|^{2} \tilde{T}(k, Y),
\end{aligned}
$$

where now the wave function of the photon is expressed in momentum space and $\tilde{T}(k, Y)$ is the scattering amplitude in momentum space. The AGBS model analytically interpolates between the behaviors of the BK scattering amplitude in the dilute regime, which is described by (12), and the saturation one, in which it behaves like

$\tilde{T}(k, Y)(k) \stackrel{k \ll Q_{\mathrm{s}}}{=} c-\log \left(\frac{k}{Q_{\mathrm{s}}(Y)}\right)$.

If one defines the variable $\rho \equiv \ln \left(k^{2} / k_{0}^{2}\right)$, the interpolation in the AGBS model is done through the following expression for the scattering amplitude:

$\tilde{T}^{\mathrm{AGBS}}(\rho, Y)=L_{F}\left(1-\mathrm{e}^{-T_{\mathrm{dil}}}\right)$,

where

$T_{\mathrm{dil}}=\exp \left[-\gamma_{\mathrm{c}}\left(\rho-\rho_{\mathrm{s}}\right)-\frac{\mathcal{L}^{2}-\log ^{2}(2)}{2 \bar{\alpha} \chi^{\prime \prime}\left(\gamma_{\mathrm{c}}\right) Y}\right]$

$\mathcal{L}=\ln \left[1+\mathrm{e}^{\left(\rho-\rho_{\mathrm{s}}\right)}\right] \quad$ with $Q_{\mathrm{s}}^{2}(Y)=k_{0}^{2} \mathrm{e}^{\lambda Y}$,

and

$L_{F}=1+\ln \left[\mathrm{e}^{\frac{1}{2}\left(\rho-\rho_{\mathrm{s}}\right)}+\mathrm{e}^{-\frac{1}{2}\left(\rho-\rho_{\mathrm{s}}\right)}\right]$.

Through this parametrization, the measurements for the $F_{2}$ structure function were successfully reproduced with the contributions of light and heavy (charm) quarks included in the fit.

To include the fluctuations in the description of the HERA data, one considers the scattering amplitude given by the AGBS model as a single event one, and one has to evaluate the average scattering amplitude, which is obtained by performing the integration

$\left\langle\tilde{T}_{Y}^{\mathrm{AGBS}}\left(\rho,\left\langle\rho_{\mathrm{s}}\right\rangle\right)\right\rangle=\int_{-\infty}^{+\infty} \mathrm{d} \rho_{\mathrm{s}} P_{Y}\left(\rho_{\mathrm{s}}\right) \tilde{T}_{Y}^{\mathrm{AGBS}}\left(\rho, \rho_{\mathrm{s}}\right)$.

This is the expression which must be inserted into (22) in order to reproduce the DIS measurements of the structure function $F_{2}$ with fluctuations effects correctly included. 


\section{Data set and results}

In this analysis, all the last HERA data measurements of the proton structure function from the $\mathrm{H} 1$ and ZEUS Collaborations [29-31] are fitted, within the following kinematical range:

$x \leq 0.01$,

$0.045 \leq Q^{2} \leq 150 \mathrm{GeV}^{2}$,

which corresponds to 279 data points. Both ranges include values of $x$ low enough for the analysis to be in the high energy regime, and values of $Q^{2}$ which allow us not to include DGLAP corrections.

Figures 1 and 2 show the structure function $F_{2}$ in bins of $Q^{2}$ for small and moderate values of $Q^{2}$, respectively. As usual, the $\mathrm{H} 1$ data have been rescaled by a factor 1.05 which is within the normalization uncertainty. Tables 1 and 2 show the values of the parameters obtained from the fit both with and without fluctuations. The latter corresponds to the value $D=0$ for the diffusion coefficient.

Concerning the parameters, we keep $\bar{\alpha}=0.2$ fixed, which enters into the amplitude through (13), and $\gamma_{\mathrm{c}}=0.6275$, whose value corresponds to the one obtained from the LO BFKL kernel. The other parameters in the amplitude, $\lambda, k_{0}^{2}$ and $\chi^{\prime \prime}$, are left free, as well as the proton radius $R_{p}$, which fixes the normalization of the dipole-proton cross section with respect to the dipole-proton amplitude, and the diffusion coefficient $D$. Only light quarks are considered and the values used for their masses are $m_{u, d, s}=50$ and $140 \mathrm{MeV}$.

\section{Conclusions and discussion}

In this paper, the AGBS model is used to investigate the possible effects of the gluon number fluctuations in the HERA data. The expression for the amplitude, (24), is considered as a single event amplitude and the average amplitude is evaluated through (28) and put into the expression for the structure function of the proton, whose data were successfully reproduced. This is shown by the good $\chi^{2} /$ n.o.p. and by the curves in Figs. 1 and 2. From the comparison between the results with and without fluctuations (see Tables 1 and 2) one sees that the value of the $\chi^{2} /$ n.o.p. does not change, and the same can be said for the parameters. Specially for the diffusion coefficient $D$, its value obtained in the case with fluctuations is very small, actually very near its mean-field value $D=0$. Then in the framework of the AGBS model, there is no evidence of fluctuations in the DIS experiment at HERA energies. This indicates that a mean-field treatment, with fixed coupling, is enough to investigate high energy QCD phenomenology, at least at HERA energies.

It is interesting to compare our results with those obtained in [14], where the GBW and IIM models are used to parametrize the average dipole-proton scattering the amplitude. Although this analysis results in a successful description of HERA data, it is not conclusive on the presence of fluctuations. In particular, the value of $D$ extracted from the fit agrees with the values predicted in the literature $[32,33]$, i.e., a sizable number of order $\mathcal{O}(1)$, which would indicate that fluctuations could be present at HERA. However, one should point out that in [14], the fit is performed using only data from the ZEUS Collaboration $[30,31]$, i.e., it does not include the H1 data [29], and within a more re-
Fig. 1 Predictions for the $\mathrm{H} 1$ [29] and ZEUS [30, 31] data for the proton structure function versus $x$ for small values of $Q^{2}$, given in $\mathrm{GeV}^{2}$. The fit was performed with quark masses $m_{u, d, s}=140 \mathrm{MeV}$

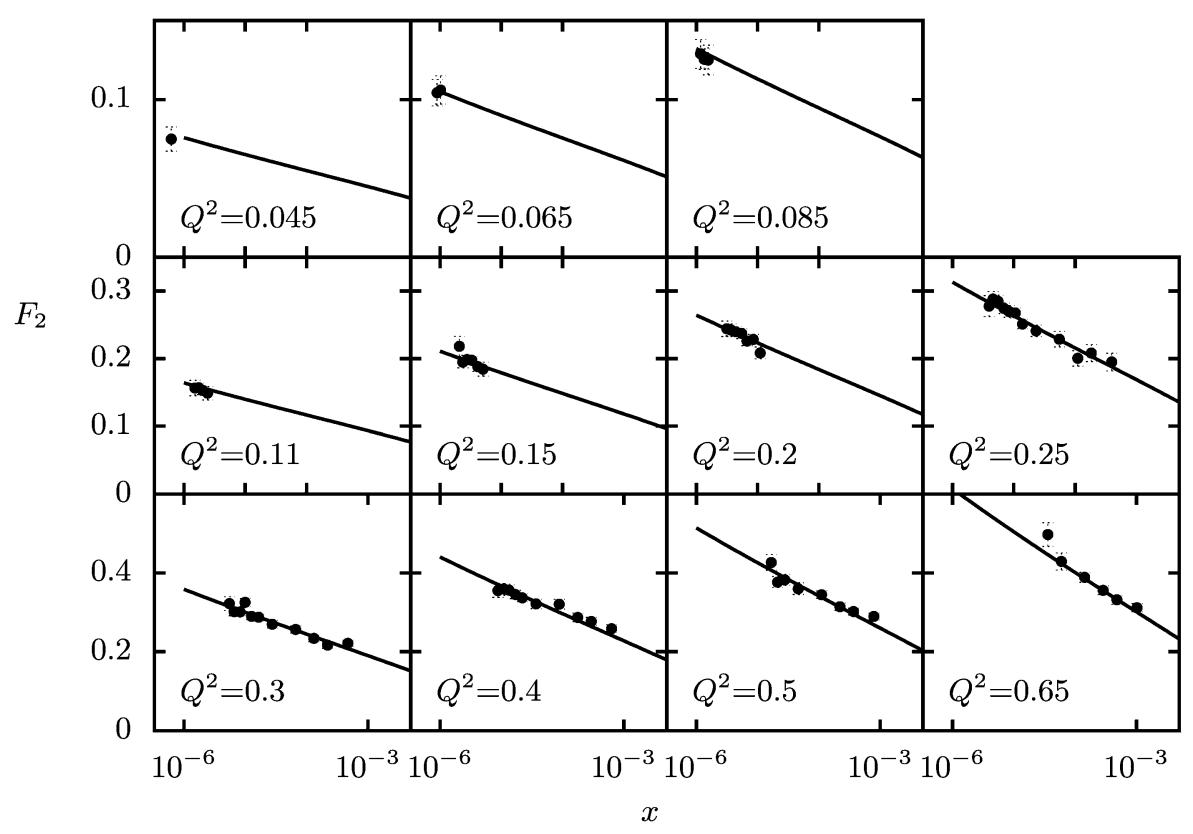


Fig. 2 Predictions for the $\mathrm{H} 1$ [29] and ZEUS [30, 31] data for the proton structure function versus $x$ for moderate values of $Q^{2}$, given in $\mathrm{GeV}^{2}$. The fit was performed with quark masses $m_{u, d, s}=140 \mathrm{MeV}$

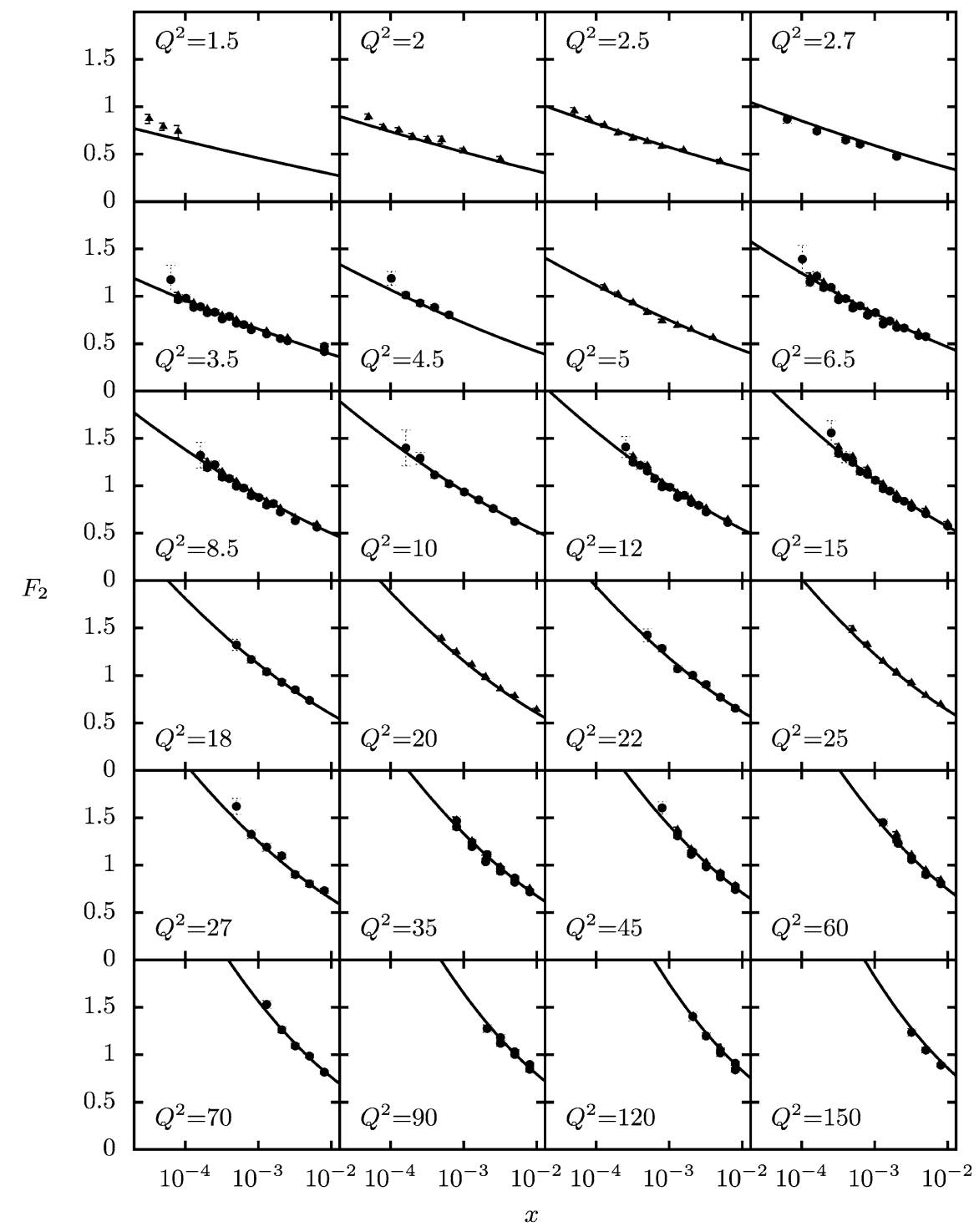

Table 1 Parameters extracted from the fit to $F_{2} \mathrm{H} 1$ and ZEUS data [29-31] in the case that $m_{u, d, s}=50 \mathrm{MeV}$

\begin{tabular}{lllllll}
\hline & $\chi^{2} /$ n.o.p. & $k_{0}^{2}\left(\times 10^{-3}\right)$ & $\lambda$ & $R\left(\mathrm{GeV}^{-1}\right)$ & $\chi^{\prime \prime}\left(\gamma_{\mathrm{c}}\right)$ & $D\left(\times 10^{-2}\right)$ \\
\hline$\tilde{T}_{Y}^{\mathrm{AGBS}}$ & 0.949 & $3.79 \pm 0.30$ & $0.213 \pm 0.003$ & $3.576 \pm 0.059$ & $4.69 \pm 0.23$ & 0 \\
$\left\langle\tilde{T}_{Y}^{\mathrm{AGBS}}\right\rangle$ & 0.949 & $3.79 \pm 0.30$ & $0.213 \pm 0.003$ & $3.576 \pm 0.059$ & $4.69 \pm 0.23$ & $0.0 \pm 1.1$ \\
\hline
\end{tabular}

Table 2 Parameters extracted from the fit to $F_{2} \mathrm{H} 1$ and ZEUS data [29-31] in the case that $m_{u, d, s}=140 \mathrm{MeV}$

\begin{tabular}{lllllll}
\hline & $\chi^{2} /$ n.o.p. & $k_{0}^{2}\left(\times 10^{-3}\right)$ & $\lambda$ & $R\left(\mathrm{GeV}^{-1}\right)$ & $\chi^{\prime \prime}\left(\gamma_{\mathrm{c}}\right)$ & $D\left(\times 10^{-3}\right)$ \\
\hline$\tilde{T}_{Y}^{\mathrm{AGBS}}$ & 0.942 & $1.69 \pm 0.16$ & $0.176 \pm 0.004$ & $4.83 \pm 0.12$ & $6.43 \pm 0.29$ & 0 \\
$\left\langle\tilde{T}_{Y}^{\mathrm{AGBS}}\right\rangle$ & 0.942 & $1.69 \pm 0.16$ & $0.176 \pm 0.004$ & $4.83 \pm 0.12$ & $6.43 \pm 0.29$ & $0.0 \pm 9.6$ \\
\hline
\end{tabular}

Table 3 Parameters extracted from the fit to $F_{2}$ ZEUS data $[30,31]$ in the case that $m_{u, d, s}=50 \mathrm{MeV}$

\begin{tabular}{lllllll}
\hline & $\chi^{2} /$ n.o.p. & $k_{0}^{2}\left(\times 10^{-3}\right)$ & $\lambda$ & $R\left(\mathrm{GeV}^{-1}\right)$ & $\chi^{\prime \prime}\left(\gamma_{\mathrm{c}}\right)$ & $D$ \\
\hline$\tilde{T}_{Y}^{\mathrm{AGBS}}$ & 0.788 & $4.26 \pm 0.43$ & $0.214 \pm 0.005$ & $3.497 \pm 0.068$ & $4.34 \pm 0.28$ \\
$\left\langle\tilde{T}_{Y}^{\mathrm{AGBS}}\right\rangle$ & 0.782 & $4.02 \pm 0.56$ & $0.190 \pm 0.030$ & $3.64 \pm 0.21$ & $3.84 \pm 0.21$ & $0.9 \pm 1.2$ \\
\hline
\end{tabular}


Table 4 Parameters extracted from the fit to $F_{2}$ ZEUS data $[30,31]$ in the case that $m_{u, d, s}=140 \mathrm{MeV}$

\begin{tabular}{lllllll}
\hline & $\chi^{2} /$ n.o.p. & $k_{0}^{2}\left(\times 10^{-3}\right)$ & $\lambda$ & $R\left(\mathrm{GeV}^{-1}\right)$ & $\chi^{\prime \prime}\left(\gamma_{\mathrm{c}}\right)$ & $D$ \\
\hline$\tilde{T}_{Y}^{\mathrm{AGBS}}$ & 0.778 & $1.97 \pm 0.22$ & $0.177 \pm 0.006$ & $4.68 \pm 0.14$ & $5.95 \pm 0.94$ \\
$\left\langle\tilde{T}_{Y}^{\mathrm{AGBS}}\right\rangle$ & 0.768 & $1.38 \pm 0.12$ & $0.120 \pm 0.010$ & $5.459 \pm 0.043$ & $5.46 \pm 0.55$ & $1.78 \pm 0.38$ \\
\hline
\end{tabular}

stricted kinematical range, with values of virtuality such that $Q_{\max }^{2}=50 \mathrm{GeV}^{2}$.

Actually, if one performs the analysis as in [14]—only ZEUS in the same kinematical range-using the AGBS model, the results obtained are very similar, as can be seen in Tables 3 and 4, with a better $\chi^{2} /$ n.o.p. The present analysis is more complete, since it includes all the last HERA data and considers a wider kinematical range.

Our conclusions seem to shed some light on the investigation of the effects of fluctuations at HERA and confirm the robustness of the AGBS model. Of course, only in the near future, at LHC, it will be possible to see if fluctuations are present at much higher energies, or if they are really suppressed by the running of the coupling, as has been suggested by recent developments on a one-dimensional toy model which successfully reproduces the main features of scattering and high energy evolution in QCD [33, 34].

Acknowledgement This work is partially supported by CNPq.

\section{References}

1. E. Iancu, A.H. Mueller, S. Munier, Phys. Lett. B 606, 342 (2005)

2. E. Iancu, D.N. Triantafyllopoulos, Nucl. Phys. A 756, 419 (2005)

3. E. Iancu, A.H. Mueller, Nucl. Phys. A 730, 494 (2004)

4. A.H. Mueller, A.I. Shoshi, Nucl. Phys. B 692, 175 (2004)

5. I. Balitsky, Nucl. Phys. B 463, 99 (1996). arXiv:hep-ph/9509348

6. Y.V. Kovchegov, Phys. Rev. D 60, 034008 (1999). arXiv:hep-ph/ 9901281

7. Y.V. Kovchegov, Phys. Rev. D 61, 074018 (2000). arXiv:hep-ph/ 9905214

8. A.M. Stasto, K. Golec-Biernat, J. Kwiecinski, Phys. Rev. Lett. 86, 596 (2001). arXiv:hep-ph/0007192

9. S. Munier, R. Peschanski, Phys. Rev. Lett. 91, 232001 (2003). arXiv:hep-ph/0309177

10. S. Munier, R. Peschanski, Phys. Rev. D 69, 034008 (2004). arXiv:hep-ph/0310357
11. S. Munier, R. Peschanski, Phys. Rev. D 70, 077503 (2004). arXiv:hep-ph/0310357

12. K. Golec-Biernat, M. Wusthoff, Phys. Rev. D 59, 014017 (1999). arXiv:hep-ph/9807513

13. E. Iancu, K. Itakura, S. Munier, Phys. Lett. B 590, 199 (2004). arXiv:hep-ph/0310338

14. M. Kozlov, A. Shoshi, W. Xiang, J. High Energy Phys. 0710, 020 (2007). arXiv:0707.4142 [hep-ph]

15. J.T. de Santana Amaral, M.B. Gay Ducati, M.A. Betemps, G. Soyez, Phys. Rev. D 76, 094018 (2007). arXiv:hep-ph/ 0612091

16. A.H. Mueller, Nucl. Phys. B 415, 373 (1994)

17. A.H. Mueller, B. Patel, Nucl. Phys. B 425, 471 (1994)

18. A.H. Mueller, Nucl. Phys. B 437, 107 (1995)

19. J. Jalilian-Marian, A. Kovner, A. Leonidov, H. Weigert, Nucl. Phys. B 504, 415 (1997). arXiv:hep-ph/9701284

20. E. Iancu, A. Leonidov, L.D. McLerran, Nucl. Phys. A 692, 583 (2001). arXiv:hep-ph/0011241

21. H. Weigert, Nucl. Phys. A 703, 823 (2002). arXiv:hep-ph/ 0004044

22. L.N. Lipatov, Sov. J. Nucl. Phys. 23, 338 (1976)

23. E.A. Kuraev, L.N. Lipatov, V.S. Fadin, Sov. Phys. J. Exp. Theor. Phys. 45, 199 (1977)

24. I.I. Balitsky, L.N. Lipatov, Sov. J. Nucl. Phys. 28, 822 (1978)

25. R.A. Fisher, Ann. Eugen. 7, 355 (1937)

26. A. Kolmogorov, I. Petrovsky, N. Piscounov, Mosc. Univ. Bull. Math. A 1, 1 (1937)

27. C. Marquet, G. Soyez, B.W. Xiao, Phys. Lett. B 639, 635 (2006)

28. Y. Hatta, E. Iancu, C. Marquet, G. Soyez, D.N. Triantafyllopoulos, Nucl. Phys. A 773, 95 (2006)

29. C. Adloff et al. [H1 Collaboration], Eur. Phys. J. C 21, 33 (2001). arXiv:hep-ex/0012053

30. J. Breitweg et al. [ZEUS Collaboration], Phys. Lett. B 487, 273 (2000). arXiv:hep-ex/0006013

31. S. Chekanov et al. [ZEUS Collaboration], Eur. Phys. J. C 21, 443 (2001). arXiv:hep-ex/0105090

32. G. Soyez, Phys. Rev. D 72, 016007 (2005)

33. E. Iancu, J.T. de Santana Amaral, G. Soyez, D.N. Triantafyllopoulos, Nucl. Phys. A 786, 131 (2007)

34. A. Dumitru, E. Iancu, L. Portugal, G. Soyez, D.N. Triantafyllopoulos, J. High Energy Phys. 0708, 062 (2007) 